\title{
Impact of mucoadhesive polymeric nanoparticulate systems on oral bioavailability of a macromolecular model drug
}

Angela Fabiano ${ }^{\mathrm{a}}$; Anna Maria Piras ${ }^{\mathrm{a}}$; Gloria Uccello-Barretta ${ }^{\mathrm{b}}$; Federica Balzano ${ }^{\mathrm{b}}$; Andrea Cesari ${ }^{\text {b; }}$ Lara Testai ${ }^{\text {a }}$, Valentina Citia; Ylenia Zambito ${ }^{\text {a* }}$

${ }^{a}$ Department of Pharmacy, University of Pisa, Via Bonanno 33, 56126 Pisa, Italy

${ }^{b}$ Department of Chemistry and Industrial Chemistry, University of Pisa, UdR INSTM Pisa, Via Moruzzi 13, 56124 Pisa, Italy

Corresponding author:

Ylenia Zambito

Department of Pharmacy, University of Pisa, Via Bonanno 33, 56126 Pisa, Italy e-mail: ylenia.zambito@unipi.it

Tel/fax: + $390502219657 / 0502219660$ 


\begin{abstract}
Nanoparticles (NP) only different in mucoadhesivity are compared for impact on drug oral bioavailability. Two polymeric NP types based on quaternary ammonium-chitosan (NP QA-Ch) and S-protected thiolated derivative thereof (NP QA-Ch-S-pro), respectively, containing the macromolecular drug model, FD4, were prepared by crosslinking each polymer with reduced MW hyaluronic acid. The structure of basic polymers was determined by $\mathrm{H}^{1} \mathrm{NMR}$ analysis. NP were similar in size ( $371 \pm 38$ vs. $376 \pm 82 \mathrm{~nm})$; polydispersity index $(0.39 \pm 0.08$ vs. $0.41 \pm 0.10)$; zeta potential $(13.4 \pm 0.9$ vs. $11.9 \pm 1.2 \mathrm{mV})$; reversible interactions with drug (bound drug, 67 vs. $66 \%$ ); encapsulation efficiency ( $23 \pm 5$ vs. $23 \pm 8 \%$ ); release properties (15\% released in $15 \mathrm{~h}$ in both cases); and apparent permeation across excised rat intestine $\left(\mathrm{P}_{\text {app }}, 8.8 \pm 0.8\right.$ vs. $\left.10 \pm 1 \mathrm{~cm} / \mathrm{s}\right)$. Then the differences in $\mathrm{NP}$ transport ratio through mucus (TR, 0.75 vs. 0.37 ) and adhesion to excised rat intestinal mucosa (adsorbed fraction, $23 \pm 3$ vs. $45 \pm 2 \%$ ) were ascribed to higher mucoadhesivity of NP QA-Ch-S-pro compared to NP QA-Ch. This directly influenced drug oral bioavailability in rats ( $\mathrm{T}_{\max }, 1 \mathrm{vs.} 2 \mathrm{~h}$; AUC, $1.7 \pm 0.3 \mathrm{vs}$. $2.9 \pm 0.4 \mu \mathrm{g} / \mathrm{mL} \min$, for NP QA-Ch and NP QA-Ch-S-pro, respectively). Mucoadhesivity increases drug bioavailability by retaining NP at its absorption site and opposing its transit down the GI tract. Data on drug accumulation in rat liver allows the assertion that NP is absorbed by transcytosis across intestinal epithelium and transported from blood into liver by Kuppfer cells.
\end{abstract}

Keywords: mucoadhesive nanoparticles; mucus diffusivity; FD4 oral bioavailability; chitosan derivatives; multifunctional chitosan. 


\section{Introduction}

The gastrointestinal (GI) tract is the primary site of drug delivery, in fact more than $80 \%$ of drugs are taken orally. The GI tract is covered by a mucus layer, which can trap and coat foreign particulates and pathogens to protect the underlying epithelium [1]. Mucoadhesive polymeric nanoparticulate systems (NP) have raised interest as vehicles for drug delivery by the oral route for their potential ability to improve the bioavailability of drugs with low mucosal permeability and/or poor chemical stability in the gastrointestinal environment [24]. To be absorbed across the intestinal epithelium into the systemic circulation, however, the NP will have to cross the layer of stagnant mucus adjacent to the intestinal membrane. In this light, mucus represents a barrier which can affect the transit of most orally administered NP [5, 6]. Indeed, the NP tendency to adhere to the mucus gel can play an important role since on the one hand it opposes the physiologic transit of the delivery system down the GI tract away from the absorption site, thus favoring drug absorption, whereas, on the other hand, it hampers the water-driven transport of NP from luminal to epithelial side of the mucus layer, thus slowing down absorption. In fact, still open to debate is the issue of whether mucoadhesive particles prolong drug residence at the absorption site and enhance drug bioavailability [7-10] or, instead, aggregate in mucus far away from the absorptive epithelium and lower the bioavailability [11-13]. In the present work our group have taken part in the debate by comparing the ability of two NP types, apparently only different in mucoadhesivity, to promote the oral bioavailability of fluorescein isothiocianate dextran (FD4, MW $4 \mathrm{kDa}$ ). This has been used by other authors $[14,15]$ as a model of macromolecular drugs because of its molecular weight, close to those of insulin and calcitonin, as well as its water solubility and hydrophilic properties and, furthermore, because it is not susceptible to enzymatic degradation in the intestinal tract. Water-soluble quaternary ammonium-chitosan derivatives were used to prepare the NP. To differentiate between more and less mucoadhesive NP, these were prepared from a novel quaternary ammonium chitosan containing protected thiol groups on its chains and a thiol-free one. According to literature information, indeed, the former was expected to be more mucoadhesive than the latter [16]. The former, moreover, was preferred over its precursor because non-protected thiols, although favoring polymer mucoadhesion, are unstable as prone to easy oxidation. The similarity of the two NP types for particle size, polydispersity index, zeta potential, permeation across excised intestine, water-assisted transport through intestinal mucus, and in vitro drug release was verified, to rule out any effect on drug bioavailability not ascribable to the difference in NP mucoadhesivity. Finally, the NP were compared on an oral bioavailability basis using rats.

\section{Materials and methods}

\subsection{Materials}


The following materials were used, along with other usual reagent grade solvents and chemicals: fluorescein isothiocyanate dextran (MW, 4kDa; FD4), thioglycolic acid, 1-ethyl-3-(3-dimethylaminopropyil) carbodiimide hydrochloride, cellulose membrane tubing MW cut-off $12.5 \mathrm{kDa}, 6-$ chloronicotinamide, thiourea, fluorescein isothiocyanate (FITC), low molecular weight chitosan (Ch) (all from Sigma); 2-diethylaminoetihyl chloride hydrochloride (Fluka); hyaluronic acid (HA), Mw 950 kDa (Contipro, Dolní Dobrouč, Czech Republic). The above materials were used, each where appropriate, to prepare the following polymers and polymer derivatives according to the methods described in the references cited: reduced-MW hyaluronic acid (rHA, viscometric MW $470 \mathrm{kDa}$ ) [17]; quaternary ammonium-Ch conjugates synthesized at $60^{\circ} \mathrm{C}$, coded QA-Ch [18]; and thiolated derivative of QA-Ch, coded QA-Ch-SH [17].

All aqueous solutions/dispersions where prepared with freshly distilled water.

\subsection{Synthesis of S-protected derivative of QA-Ch-SH: QA-Ch-S-pro}

As 6-mercaptonicotinamide (6-MNA) is commercially not available, it was synthesized according to Forrest and Walker (1948) [19]. Thiourea and 6chloronicotinamide $(1: 1)$ was suspended in ethanol and refluxed $6 \mathrm{~h}$ under nitrogen. Overtime, the mixture turned light yellow and was allowed to cool down to room temperature and stirred overnight. The resulting salt, S-(5carbamyl-2-pyridyl)-thiouronium chloride, was separated by filtration and dried. The dried thiouronium salt was decomposed by adding $2 \mathrm{~N} \mathrm{NaOH}$ and stirring $45 \mathrm{~min}$ at room temperature. The $\mathrm{pH}$ was adjusted to 4 with acetic acid and the light yellow suspension turned to dark yellow. The resulting 6-MNA was isolated by filtration, washed with water and dried. Next, it was suspended in water and the $\mathrm{pH}$ adjusted to 7 , after which a hydrogen peroxide solution was added. The mixture was stirred $1 \mathrm{~h}$ at room temperature, whereby the yellow color turned back to white. The resulting 6,6'-dithionicotinamide (6,6'-DTNA) was isolated by filtration, washed with water and dried. The covalent attachment of the aromatic ligand to QA-Ch-SH was achieved by disulfide bond formation between thiol groups of 6-MNA and free thiol groups of QA-Ch-SH according to Dünnhaupt, et al. (2012) [20] Two hundred mg of lyophilized QA-Ch-SH was dissolved in $50 \mathrm{~mL}$ of a mixture of DMSO and water (7:3). Fifty mg of 6-6'DTNA was dissolved in $50 \mathrm{ml}$ of DMSO and added to the QA-Ch-SH mixture. After addition of the ligand, the $\mathrm{pH}$ was adjusted to 6 and the reaction mixture stirred $6 \mathrm{~h}$. For purification, the mixture was dialyzed 4 days against water. After dialysis, the polymer solution was lyophilized to obtain the purified protected thiomer (QA-Ch-S-pro) (Figure 1).

\subsection{Determination of thiol content in polymers}


The quantification of free thiol moieties was performed for both QA-Ch-SH and QA-Ch-S-pro. Polymers were dissolved in $10 \mathrm{~mL}$ of water, $1 \%$ starch aqueous solution $(1 \mathrm{~mL})$ was added, the $\mathrm{pH}$ was adjusted to 3 with $1 \mathrm{M} \mathrm{HCl}$ and the solution was titrated with $1 \mathrm{mM}$ aqueous iodine until a permanent light blue discoloration was observed [21]. The quantification of degree of substitution by thiol-bearing groups in both polymers was performed after borohydride red-ox reaction. In details, $8 \mathrm{~mL}$ of $10 \%$ aqueous sodium borohydride to a solution of 15 $\mathrm{mg}$ polymer in $2 \mathrm{~mL}$ of water, and stirring for $1 \mathrm{~h}$. Then the excess of sodium borohydride was destroyed by making the solution to $\mathrm{pH} 3$ with $1 \mathrm{M} \mathrm{HCl}$ and the thiol content was determined by iodometric titration as described above.

\subsection{Quantification of the protected thiols in QA-Ch-S-pro}

Samples of $0.1 \%(\mathrm{~m} / \mathrm{v})$ polymer solution $(5 \mathrm{ml})$ were added to reduced glutathione $(0.1 \%)$ and the mixture was stirred $2 \mathrm{~h}$, after which it was analyzed spectrophotometrically at $307 \mathrm{~nm}$. A calibration curve was obtained with 6-MNA standard solutions. The content of conjugated aromatic ligand was expressed as degree of substitution (\%) on a polymer repeating unit basis of polymer.

\subsection{NMR studies}

${ }^{1} \mathrm{H}$ NMR measurements were performed in phosphate buffered $\mathrm{D}_{2} \mathrm{O}(\mathrm{pH}=7.4)$ solution on a spectrometer operating at $600 \mathrm{MHz}$. The temperature was controlled to $25 \pm 0.1^{\circ} \mathrm{C}$. ${ }^{1} \mathrm{H}$ NMR spectrum was recorded with $20 \mathrm{~s}$ relaxation delay. After data acquisition, each FID was apodized with $1.0 \mathrm{~Hz}$ line broadening and Fourier transformed. The selected signals of protective group were processed by the qEstimate software (Agilent) and by comparing its integrated area with that of a standard sample of known concentration. The 2D NMR spectra were obtained by using standard sequences. Proton gCOSY (gradient COrrelated SpectroscopY) 2D spectra were recorded in the absolute mode acquiring 64 scans with a $1 \mathrm{~s}$ relaxation delay between acquisitions and 4K data points for each of 200 FIDs. 2D TOCSY (TOtal Correlation SpectroscopY) spectrum was recorded acquiring 64 scans with a $1 \mathrm{~s}$ relaxation delay, 200 increments, $4 \mathrm{~K}$ data points and a mixing time of $0.1 \mathrm{~s}$. The gradient gHSQC (gradient Heteronuclear Single Quantum Coherence) spectra and gHMBC (gradient Heteronuclear Multiple Bond Coherence) were obtained in 256 scans and 128 scans per increment respectively The spectral width used was the minimum required in both dimensions.

\subsection{Preparation and characterization of NP}

NP based on either QA-Ch or QA-Ch-S-pro derivative was prepared by selfassembly upon addition of rHA. In details, a solution of $\mathrm{rHA} 0.2 \mathrm{mg} / \mathrm{ml}$ in

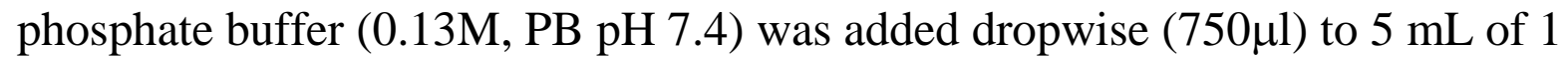
$\mathrm{mg} / \mathrm{mL}$ polymer solution in the same buffer, under stirring at room temperature. To prepare FD4-loaded NP the above rHA solution contained $3 \mathrm{mg}$ of FD4. The final FD4 concentration in the NP dispersion was $0.52 \mathrm{mg} / \mathrm{mL}$. Following 
preparation, the NP dispersion was checked for particle size and $\zeta$-potential (Beckman/Coulter N4 Plus, Beckman/Coluter Delsa ${ }^{\mathrm{TM}}$ Nano). To purify the FD4loded NP, the dispersion was centrifuged $\left(30 \mathrm{~min}\right.$ at $20,000 \mathrm{rpm}$ and $4{ }^{\circ} \mathrm{C}$, Termo Scientific MTX 150), the supernatant was removed, analyzed fluorometrically (excitation $490 \mathrm{~nm}$, emission $530 \mathrm{~nm}$, Perkin Elmer LS45) to evaluate the FD4 amount entrapped in the NP, and the sediment was re-suspended in $5 \mathrm{~mL}$ of $\mathrm{H}_{2} \mathrm{O}$ and lyophilized. The dispersions could be regenerated from the respective lyophilized products by adding $5 \mathrm{~mL}$ of water under gentle stirring. The FD4 fraction entrapped in the NP (entrapment efficiency, EE) was calculated as follows, using the appropriate calibration curve:

$\mathrm{EE}=(\mathrm{Mt}-\mathrm{Ms}) / \mathrm{Mt}$

where Mt is the total mass of FD4 used for the preparation of NP and Ms is the mass found in the supernatant. The drug concentration in the regenerated dispersion (DRD) was expressed by the following equation:

$\mathrm{DRD}=(\mathrm{Mt}-\mathrm{Ms}) / \mathrm{VRD}$

where VRD is the regenerated dispersion volume.

\subsection{Dynamic dialysis studies}

A well-known procedure and underlying theory $[22,23]$ was applied to assess the reversible drug binding by NP in fluid dispersion, at the thermostat temperature of $37{ }^{\circ} \mathrm{C}$. The dialysis membrane, permeable to the drug and impermeable to the $\mathrm{NP}$, was made of porous cellulose (cut-off $12.5 \mathrm{kDa}$ ). At $\mathrm{t}=0,5 \mathrm{~mL}$ of FD4loaded NP, regenerated after lyophilization, was introduced in the donor compartment of the cell. Sink conditions were maintained in the receiving phase (100 mL of PB pH 7.4). Drug transport across the membrane was assessed by fluorometrically analyzing the receiving phase at intervals for the drug. At the end of each run, the donor phase was withdrawn from the cell and checked for particle size. The dialysis was also carried out, using the same procedure and membrane type, for the plain drug solution (control).

\subsection{Interrupted-dialysis studies}

Interrupted-dialysis experiments were carried out to gain information on drug release from NP while concurrently checking data according to the suggestions from Zambito, et al. (2012) [24]. To this purpose, the dialysis, carried out as described in Section 2.7; was stopped after established time intervals and the drug fraction contained in each of the NP matrix, NP-dispersion medium and acceptor medium was determined, after centrifuging ( $30 \mathrm{~min}$ at $20,000 \mathrm{rpm}$ and $4^{\circ} \mathrm{C}$ ) the NP sample in the donor compartment. This procedure was repeated, running the dialysis for different times, to construct the plots of the drug fraction in each phase versus time.

\subsection{In vitro study of water-assisted NP transport through mucus}

Porcine intestinal mucus samples were scraped off from the small intestinal tissues of 3 freshly slaughtered pigs, provided by a local slaughter house, and 
pooled together. The mucus was purified and homogenized, and transport studies across a purified mucus layer were carried out as described by Fabiano et al. (2017) [25]. A mucus layer was realized by filling the barrel of a $1 \mathrm{~mL}$ plastic syringe with $350 \mu \mathrm{L}$ of purified mucus. The barrel was kept, tip down, in vertical position and the mucus was prevented from escaping through the syringe tip by a cellulose nitrate $0.2 \mu \mathrm{m}$ filter. Fifty $\mu \mathrm{L}$ of NP dispersion, centrifugation-purified, lyophilized and regenerated as described in section 2.6, was placed on the filter before adding the mucus. A bottom-to-surface phosphate buffered saline $\mathrm{pH} 6.8$, $0.13 \mathrm{M}$ (isotonic) (PBS), flow across the mucus layer (diameter, $0.6 \mathrm{~cm}$ ) was realized by connecting the tip of the syringe to the bottom of a vertical cylindrical vessel by a flexible tubing, filling the vessel with PBS, level with the surface of the mucus layer in the syringe, and dripping PBS into the vessel so as to create a supernatant liquid phase gradually rising over the mucus layer at a rate of $1.24 \cdot 10^{-4} \mathrm{~cm} / \mathrm{sec}$ corresponding to a flux of $0.446 \mathrm{~mL} /\left(\mathrm{cm}^{2} \mathrm{~h}\right)$, without causing any phase mixing. Such a flux value was calculated from the data reported by Lennernans et al. (1994) for intestinal water absorption in man [26]. The system was maintained in vertical position at $37^{\circ} \mathrm{C}$ for $5 \mathrm{~h}$, after which the supernatant was withdrawn from the mucus, the syringe removed from the assembly, frozen and cut into slices of $2 \mathrm{~mm}$ length, starting from the tip where the formulations had been added. The FD4 in the supernatant was determined as already described. For determination of the FD4 in each slice the same procedure was applied after thawing out the frozen slice and removing the plastic debris.

\subsection{Ex-vivo studies}

The intestinal mucosa was excised from non-fasting male Wistar rats weighing $250-300 \mathrm{~g}$. After sacrificing the rats, the first $50 \mathrm{~cm}$ of small intestine was immediately removed and used for the ex-vivo studies. To check the tissue integrity, the electrical resistance of an excised intestine sample was determined under the start and end conditions of a permeation experiment, as described by Heinen et al., 2013 [27].

\subsubsection{Ex-vivo mucoadhesivity studies}

A previously described procedure was followed [17]. The intestinal mucosa was excised from non-fasting male Wistar rats weighing 250-300g. After sacrificing the rats, the first $50 \mathrm{~cm}$ of small intestine was immediately removed. Four $10-\mathrm{cm}$ segments were cut out of the excised intestine, opened by longitudinal cutting and the mucosa gently rinsed free of luminal content. Each segment was then immersed in $1 \mathrm{~mL}$ of the regenerated FD4-loaded NP dispersions. Incubation at $37{ }^{\circ} \mathrm{C}$, under bubbling of a mixture of $95 \% \mathrm{O}_{2}$ and $5 \%$ of $\mathrm{CO}_{2}$, lasted $4 \mathrm{~h}$ with no damage to the mucosa [28]. Then, $50 \mu \mathrm{L}$ was withdrawn, appropriately diluted and analyzed fluorometrically with reference to the relative calibration curve. The adsorbed mass fraction, listed in Table 3, was calculated from the difference between the FD4 concentration determined before and after incubation. 


\subsubsection{Permeation experiments}

A previously described procedure was followed [28]. The excised intestine was cut into strips of $1.5 \mathrm{~cm}$, rinsed free of luminal contents and mounted in Ussing type chambers $\left(0.78 \mathrm{~cm}^{2}\right.$ exposed surface area) without stripping off the underlying muscle layer. One and $3 \mathrm{ml}$ of phosphate buffer $\mathrm{pH} 7.4,0.13 \mathrm{M}$, isosmotic (PB 7.4) were added to the mucosal (donor) and serosal (acceptor) compartment, respectively, of the permeation cell. In order to ensure oxygenation and agitation, a mixture of $95 \% \mathrm{O}_{2}$ and $5 \% \mathrm{CO}_{2}$ was bubbled through each compartment. The Ussing chamber was then placed in a thermostatic water bath at $37{ }^{\circ} \mathrm{C}$. After 20 min equilibration the medium in the donor compartment was replaced with $1 \mathrm{~mL}$ of each regenerated $\mathrm{NP}$ dispersion, or a FD4 solution (control). The apical to basolateral transport of FD4 was investigated, i.e., at 30 min intervals, of a total of $240 \mathrm{~min}, 1 \mathrm{~mL}$ volume was withdrawn from the acceptor compartment and replaced with an equal volume of fresh preequilibrated PB 7.4.

For each permeation run a value of apparent permeability coefficient, $\mathrm{P}_{\text {app }}$, for permeant across the excised rat intestinal mucosa was calculated from the following equation:

$\mathrm{P}^{\prime}{ }_{\text {app }}=\mathrm{dM} / \mathrm{dt} 1 /\left(\mathrm{AC}_{0}\right)$

where $\mathrm{dM} / \mathrm{dt}$ 1/A, the permeation flux, is the slope of the linear portion of the cumulative amount permeated per unit surface area vs time plot, and $\mathrm{C}_{0}$ is the FD4 concentration introduced into the donor phase, i.e., the drug concentration in the regenerated dispersion $\left(\mathrm{C}_{0}=\mathrm{DRD}, 0.14 \mathrm{mg} / \mathrm{mL}\right)$. For each plot, the linear regression analysis was extended to the set of data points that gave the best fit, as judged from the $\mathrm{r}^{2}$ value. This, in all of the cases investigated, was $>0.9$. The single $\mathrm{P}_{\text {app }}$ values were averaged to calculate the mean apparent permeability $\mathrm{P}_{\text {app }}$ $(n, 3-6)$. The mean cumulative amount permeated per unit area in any given time was calculated to plot each permeation profile and determine $\mathrm{T}_{4 \mathrm{~h}}$, i.e., the cumulative transport per unit area over the whole time of experiment. The significance of the differences between each of the $\mathrm{P}_{\text {app }}$ or $\mathrm{T}_{4 \mathrm{~h}}$ values was assessed by the Student's $t$-test $(\mathrm{P}<0.05)$. For NP that produced a significant $\mathrm{P}_{\text {app }}$ increase, this was measured by the enhanced permeability ratio (EPR), defined as the ratio between each of the $\mathrm{P}_{\text {app }}$ values obtained with the NP and that obtained with the control plain FD4 PB solution.

\subsection{Bioavailability studies}

To evaluate the time-course of the plasma levels of FD4 twelve male Wistar rats (260-350 g) were anesthetized with thiopental (70 mg/kg, i.p.), following the guidelines from the European Community Council Directive 2010/63, approved by the Animal Care Committee of the University of Pisa (D.L. 2014/26). The trachea was intubated and connected to a rodent ventilator (mod 7025 Ugo Basile, Comerio, Italy) for artificial ventilation with room air (stroke volume, $1 \mathrm{ml} / 100 \mathrm{~g}$ body weight; 70 strokes/min). Electrocardiogram (ECG) was continuously measured by lead II (Mindray, PM5000; 2 Biological Instruments, Varese, Italy), 
in order to monitor the main vital functions. After a stabilization time of $15 \mathrm{~min}$ a blood sample $(500 \mu \mathrm{L})$ was collected (basal control). Then the animals were treated by gavage with about $400 \mu \mathrm{l}$ of FD $4(0.75 \mathrm{mg} / \mathrm{Kg})$ contained in the samples under study (i.e. plain FD4 (control), NP QA-Ch or NP QA-Ch-S-pro). Blood samples $(500 \mu \mathrm{L})$ were collected by carotid catheterization, after 30, 60, 120, 180 and $300 \mathrm{~min}$ from administration. After collection, each blood sample was immediately centrifuged $\left(2040 \mathrm{rpm}, 20 \mathrm{~min}, 4{ }^{\circ} \mathrm{C}\right)$ and the serum separated $(150 \mu \mathrm{L})$ and analyzed for FD4 fluorometrically. The area under the curve was calculated by the linear trapezoidal rule between time 0 and $300 \mathrm{~min}$.

Significance of differences was evaluated by the Student's $t$-test.

After completion of the bioavailability study the rat was sacrificed, and the liver, spleen, and kidney were withdrawn and homogenized with PBS, 10 min at 8000 rpm (Ultra-Turrax T25). The homogenate was centrifuged $5 \mathrm{~min}$ at 3,000 rpm and the supernatant $(150 \mu \mathrm{L})$ was mixed with $50 \%$ trichloroacetic acid $(30 \mu \mathrm{L})$. The mixture was centrifuged $5 \mathrm{~min}$ at $10,000 \mathrm{rpm}$ to remove the precipitated. Then the supernatant was analyzed for FD4 content fluorometrically [29]. For reference the above procedure was repeated several times on liver, spleen, and kidney of rats not treated with any of the NP dispersions. The differences were considered significant when the fluorescence value was double that of the untreated tissue value.

\subsection{Statistical data treatment}

Experiments were replicated $(n=3-6)$, the results were averaged and the statistical significance of differences between means was assessed by the Student's $t$ test. Values of $p<0.05$ were deemed sufficiently low as to reject the null hypothesis and take the differences as significant. It should be stressed that $p$-values lower than the 0.05 threshold indicate no more than a misfit of the null hypothesis to the data being compared.

\section{Results}

\subsection{Characterization of QA-Ch-SH and QA-Ch-S-pro}

The S-protected derivative QA-Ch-S-pro was obtained from its precursor QA-ChSH by disulfide bond formation with 6-MNA, through reaction with 6-6'-DTNA. The determination of the protective group content in the polymer was preceded by the characterization of the quaternary ammonium chitosan that had been employed as the starting material for the protection reaction. The ${ }^{1} \mathrm{H}$ NMR spectrum of the latter polymer, seen in Figure 2, shows five distinct spectral regions corresponding to methyl protons of ethyl moieties bound to neutral nitrogens $(0.9 \div 1.0 \mathrm{ppm})$, methyl protons of ethyl groups bound to quaternized nitrogens $(1.1 \div 1.4 \mathrm{ppm})$, methylene protons of ethyl moieties, both neutral and quaternized, superimposed on ring protons $(2.3 \div 4.1 \mathrm{ppm})$, anomeric protons as a 
broad signal in the range $4.3 \div 4.5 \mathrm{ppm}$ and, finally, residual $\mathrm{N}$-acetyl functions at $1.93 \mathrm{ppm}$.

The integrations of the different spectral regions, following a well consolidated analytical protocol [30], allowed determination of the degree of acetylation $(14.1 \%)$, degree of quaternary ammonium substitution $(63.6 \%)$ and ratio of quaternized to neutral nitrogens $(\approx 2.0)$. The ${ }^{1} \mathrm{H}$ NMR spectrum of the thiolated derivative is completely superimposable on that of the parent compound, because of a very low content in thiol groups in the former and the hardly detectable $\mathrm{SH}$ group resonance, due to the $\mathrm{H}-\mathrm{D}$ exchange in $\mathrm{D}_{2} \mathrm{O}$. Therefore, the quantitative analysis of thiol groups was performed by iodometry.

The NMR spectrum of 6-MNA-protected polymer shows an analogous pattern of signals between $0.6 \div 5 \mathrm{ppm}$, with the presence of low intensity resonances between $7.2 \div 9.0 \mathrm{ppm}$ (Figure 3) attributable to pyridyl protons.

The absence of unbound 6-MNA moieties was demonstrated by comparison with the NMR spectra of 6,6'-DTNA and all of its precursors (Fig. S1-4). 2D TOCSY map (Fig. S5) of the protected polymer demonstrated the presence of three major components (A, B and C) of bound 6-MNA. The use of a 6-MNA external standard for the quantitative analysis allowed the assessment of a $1.87 \%$ 6-MNA groups on polymer repeating unit basis, which resulted from comparing the integrated area of the proton unit of the standard with that of pyridyl protons of the polymer in NMR spectra recorded with identical spectral parameters and in quantitative conditions.

The quantitative determination of thiol content was performed iodometrically. As reported in Table 1, free thiols content of QA-Ch-S-pro was 2-fold lower than that of QA-Ch-SH, accordingly with the formation of disulphide bridges with 6MNA. This data completely agrees with that determined by NMR spectrometry.

\subsection{Characteristics of NP}

The NP size, polydispersity index, zeta potential $(\zeta)$ and encapsulation efficiency (EE) are found in Table 2. The size and polydispersity index values are not significantly different from each other and are comprised within the ranges accepted for pharmaceutical nanoparticulate systems [31]. The $\zeta$ values are positive in agreement with the presence of quaternary ammonium ions on the NP surface, and the EE is always around $20 \%$ with no significant differences between the two cases.

\subsection{Drug-binding studies}

Dynamic dialysis data were used to compare reversible drug interactions with the NP in fluid dispersions. The data obtained, according to the established theory on determination of drug-macromolecule reversible binding by dynamic dialysis, was plotted according to the following known equation [23, 32]

$\ln \left[\left(\mathrm{C}_{\mathrm{d}} / \mathrm{C}_{\mathrm{d} 0}\right) \times 100\right]=4.605-\mathrm{K}_{\mathrm{m}} \mathrm{F}_{\mathrm{f}} \mathrm{t}$

where $\mathrm{C}_{\mathrm{d}}$ is the drug concentration in donor phase, $\mathrm{C}_{\mathrm{d} 0}$ is the drug concentration in donor phase at time $\mathrm{t}=0, \mathrm{~F}_{\mathrm{f}}$ is the non interacting-to-total drug ratio in donor 
phase, and $\mathrm{K}_{\mathrm{m}}$ is the dialysis-rate constant. Once $\mathrm{F}_{\mathrm{f}}$ is determined from the slope of the log-linear plot, the bound fraction can readily be calculated as $\left(1-\mathrm{F}_{\mathrm{f}}\right) \times 100$. The dialysis data for plain FD4 (control) and FD4-loaded NP are plotted in Figure 4 as $\ln \left(\mathrm{C}_{\mathrm{d}} / \mathrm{C}_{\mathrm{d} 0}\right) \times 100$ versus $\mathrm{t}$. All plots are significantly linear, as shown by the respective $\mathrm{r}^{2}$ values, namely $0.993,0.969$ and 0.955 . Such a linearity indicates that in all cases Equation 2 is obeyed and $F_{f}$ was actually constant with varying $C_{d}$ over the time of dialysis. For the plain drug (control), according to Equation 2, the slope of the relative log-linear plot is equal to the dialysis-rate constant $\mathrm{K}_{\mathrm{m}}$. The slopes of the straight lines \pm standard deviation were $3 \pm 0.110^{2}$ hours $^{-1}$ (control), 1.0 $\pm 0.110^{2}$ hours $^{-1}$ (NP QA-Ch) and 1.0 $\pm 0.110^{2}$ hours $^{-1}$ (NP QA-Ch-Spro). These data indicate a 67.1 and $66.4 \%$ bound fraction for NP QA-Ch and NP QA-Ch-S-pro, respectively. From here, it was deduced that the drug underwent reversible interactions with both NP types with no significant difference between the two cases.

\subsection{FD4 release from NP}

The procedure used in this work was based on interrupted dialysis followed by ultracentrifugation of donor phase as described in section 2.8. Results from the kinetic analysis of phases are plotted in Figure 5.

The data in Figure 5 indicate that, for both NP types, only about $15 \%$ of the whole drug load was released from NP matrix in 15 hours, while about $85 \%$ remained entrapped in matrix. The linear regression analysis of FD4\% data for the receiving phase was significant for both NP types $\left(\mathrm{r}^{2}\right.$ values, 0.94 and 0.95 for QA-Ch in Figure 5A and QA-Ch-S-pro in Figure 5B, respectively). Considering that the FD4\% in the NP dispersion medium was virtually constant over the whole time of experiment $(24 \mathrm{~h})$, it can be stated that the membrane controlled the FD4 permeation kinetics to a pseudo-steady state. Nevertheless, as relevant data in Figure 5 show, the FD4\% in the NP dispersion medium did not substantially rise over sink conditions for FD4 release, at least within $15 \mathrm{~h}$ of experiment. Then it can be admitted that both NP types are able to substantially retain their load of the macromolecular drug model for a term sufficient for the NP to be orally absorbed.

\subsection{Transport of NP through mucus and mucoadhesivity studies}

The results of this study are represented in Figure 6. These experiments lasted 5 $h$, after which the FD4 fraction released from the NP matrix was negligible, as seen in Figure 5. At the end of the experiment, the distribution of transported products over the length of the sliced mucus layer, from slice 1 to 11 , in all cases appears substantially uniform. The fluorescence found in the supernatant is significant in all cases, indicating that all species tested were transported past slice 11. Moreover, particle size analysis showed that, in the case of NP, the fluorescence found in the supernatant was associated with the presence in it of dispersed NP. 
To bestow a quantitative significance on the results from these studies, the transport ratio (TR) was defined as the ratio between the concentration of the transported material found in supernatant in $5 \mathrm{~h}$ from the start of the experiment and that introduced at the bottom of the mucus layer at the start of it. The TR data listed in Table 3 show that the transport of NP was significantly slower than that of the FD4 control. In particular the NP QA-Ch-S-pro transport is slower than the NP QA-Ch transport, perhaps due to the presence of protected thiol groups on the polymer chain of the former. Indeed, it is known that converting thiol into disulphide groups prevents an early oxidation of the former and allows an exchange reaction of the latter with the cysteine substructures of mucus, leading to the formation of disulphide bridges between NP and mucus [33]. In this specific case, QA-Ch-S-pro carries a thiol substituent prone to an easier exchange with cysteine moieties, resulting in a stronger interaction with mucus. As a matter of fact, the adsorption of either NP type on intestinal mucus was significantly stronger than that of the control FD4 solution, as shown in Table 3. The comparison of adsorbed mass fractions in Table 3 for NP QA-Ch and NP QA-Ch-S-pro shows a significantly stronger mucoadhesivity of the latter. These data are in agreement with the corresponding TR values and indicate that the transport of NP is strongly influenced by its mucoadhesivity.

\subsection{Permeation experiments}

The excised rat jejunum was chosen for these studies, among the known ex vivo intestinal epithelium models, because its tight junctions are similar in number and tightness to those of the human jejunum [34]. The measurements of the tissue electrical resistance at the start and end of a control permeation experiment yielded values in line with literature data indicating tissue integrity [27]. A major limitation of the model ability to mimic the in-vivo absorption process mainly concerns lipophilic molecules. In fact, in the ex-vivo model the rate-limiting barrier to transport of such a molecule type is not expected to be provided by the epithelium, but rather, by the hydrophilic underlying tissue [35] This might also be the case with nanosystems transported across the epithelium by transcytosis. The apparent permeation plots for FD4, seen in Figure 7, are all significantly linear $\left(r^{2}>0,9\right)$, in agreement with our hypothesis for application of Eq.1. Such a linearity allowed the calculation of a $\mathrm{P}_{\text {app }}$ parameter for each formulation, useful for comparative purposes. The relevant data, listed in Table 4, show that both NP types had the same impact on apparent permeation parameters for FD4, in fact, no significant differences are seen between respective flux, nor $\mathrm{P}_{\text {app }}$, nor $\mathrm{T}_{4 \mathrm{~h}}$ values, all of which are significantly higher than the corresponding values for the control FD4. Since the concentration in the donor, i.e., the driving force for penetration, was the same in all cases $(0.14 \mathrm{mg} / \mathrm{mL})$ the results point to much more aptitude of the NP to cross the excised intestinal wall compared to the FD4 control.

\subsection{Bioavailability studies}


FD4 plasma concentration-time curves and corresponding pharmacokinetic parameters after NP oral administration are reported in Figure 8 and Table 5. The bioavailability of FD4 was higher when this was administered via an NP formulation. In particular, NP QA-Ch-S-pro showed a significantly higher FD4 bioavailability than NP QA-Ch. As can be seen from the pharmacokinetic data, shown in Table 5, $\mathrm{T}_{\max }$ in the case of NP QA-Ch-S-pro moves from one to two hours and this shift is accompanied by an increase in AUC. This bioavailability increase is ascribed to an increased NP residence time at the absorption site, associated with the increase in NP adhesion to the mucus lining of the intestinal epithelium. In this respect it should be considered that, as shown in our previous paper [25], NP mucoadhesivity is unable to prevent NP from crossing the mucus layer and reaching the epithelium thanks to the assistance from the lumen-toepithelium advective water movement. Figure 9 shows the FD4 amounts found in the tissues homogenized after withdrawing from rats treated with FD4 in solution, NP QA-Ch and NP QA-Ch-S-pro after 5h from administration, or untreated ones (control). No significant increase in FD4 mass in the different tissues following plain FD4 administration is observed, whereas a significant increase of such a mass in the liver corresponds to FD4 administration via NP QA-Ch or NP QACh-S-pro. The relevant enhancement factor $(\mathrm{EF})$, calculated as the ratio between the FD4 amount found in the liver after NP administration and that found in the liver of non-treated rats (control), is 1.5 with both NP types. This data points to an FD4 accumulation in liver consequent to NP administration. It was demonstrated that with varying NP size between 50 and $500 \mathrm{~nm}$, there was a higher level of accumulation of the NP in the liver [36]. This was ascribed to their greater surface area for interaction with the cell membrane and adjacent receptor. Kupffer cells recognize NP as a foreign material and the NP can be internalized through multiple scavenger receptors [37] For many applications, the interaction and removal of NP from the bloodstream by Kupffer cells are considered a significant challenge to targeting for diagnostic or therapeutic applications.

\section{Discussion}

In order to assess the impact of mucoadhesivity on NP ability to enhance the bioavailability of encapsulated drugs, NP similar in size, zeta potential, reversible interactions with the drug, drug loading and release properties ought to be compared on a mucoadhesivity basis. Therefore, the synthesis was devised of a novel S-protected thiomer, derived from quaternized chitosan, only different from the parent polymer for mucoadhesivity. Such a thiomer was obtained by applying an already described reaction to a thiolated and quaternized, in place of a simply thiolated chitosan. The resulting polymer, despite the high lipophilicity of the protecting group, was highly water-soluble irrespective of $\mathrm{pH}$, and hence, easier to handle. It was reported, indeed, that a thiolated chitosan derivative with thiols protected by a good leaving group is more mucoadhesive than the parent thiolated polymer [16]. Our goal of obtaining two NP types only different in mucoadhesivity has in fact been achieved so far as size, polydispersity index, zeta 
potential, encapsulation efficiency (shown in Table 2), drug release (seen in Figure 5), reversible drug binding (shown in Section 3.3) and ability to enhance FD4 apparent permeability across excised rat intestine $\left(\mathrm{P}_{\text {app }}\right.$, found in Table 5) of both NP types have been found not to be significantly different. The diffusion through mucus data, presented in Figure 6, have confirmed the great extent to which NP mucoadhesivity hampers NP transport through mucus. Nevertheless, our experimental model, able to simulate the lumen-to-epithelium water advective flow occurring in the human intestine, has shown that even the mucoadhesive NP can cross the mucus lining of the intestine thanks to the push from such a flow. A contribution to such an NP movement may also be given by the exchange reactions between the disulphide groups of the S-protected polymer and the thiols of the mucus glycoproteins. Probably, once a disulphide bridge between NP and mucus had formed, such a bond could break and re-form several times, because of both the water movement and the reducing conditions of the microenvironment $[1,38]$. What stated above has made the NP QA-Ch-S-pro more apt to enhance the FD4 bioavailability than the less mucoadhesive NP QA-Ch. Indeed, the difficulty encountered by the former in crossing the intestinal lining from lumen to epithelium is compensated for by its ability to oppose the physiological transit down the GI tract. Although the above results cannot be generalized they, nevertheless, along with those reported by others [7-10] prompt the notion of the importance of NP mucoadhesion in prolonging the drug residence at the absorption site, and enhancing the drug bioavailability. The drug release kinetics from NP can well be determinant to bioavailability. In fact, the active principle, especially if degradable by the enzymes of the GI tract, would not reach the systemic circulation, in case it is released during the NP transit across the mucus or, anyway, before NP internalization into the enterocytes of the intestinal epithelium. Our group have faced such a problem when dealing with the transcorneal administration of metenkephalin [39]. In the present study, both NP types released only $15 \%$ of FD4 after $15 \mathrm{~h}$ under sink conditions, therefore a significant increase of FD4 mass was found in the liver of rats treated with either NP type, as shown by the data in Figure 9.

\section{Conclusions}

These results indicate that drugs trapped in the more mucoadhesive NP type have higher oral bioavailability than those trapped in the less mucoadhesive ones. Indeed, mucoadhesivity tends to keep the formulation at the absorption site, opposing its physiologic movement from stomach down to large intestine, while the concurrent water movement facilitates NP transport across the mucus layer from lumen to epithelium, where NP can be internalized by the cells.

\section{Bibliography}

1. R.A. Cone, Barrier properties of mucus, Adv. Drug Deliv. Rev. 61 (2009) 75-85. https://doi.org/10.1016/j.addr.2008.09.008 
2. S. Dünnhaupt, J. Barthelmes, J. Hombach, D. Sakloetsakun, V. Arkhipova, A. Bernkop-Schnürch, Distribution of thiolated mucoadhesive nanoparticles on intestinal mucosa, Int. J. Pharm. 408 (2011) 191-199. https://doi.org/10.1016/j.ijpharm.2011.01.060

3. G. Ponchel, M.J. Montisci, A. Dembri, C. Durrer, D. Duchêne, Mucoadhesion of colloidal particulate systems in the gastro-intestinal tract, Eur J. Pharm. Biopharm. 44 (1997) 25-31. https://doi.org/10.1016/S09396411(97)00098-2

4. B. Sarmento, A. Ribeiro, F. Veiga, P. Sampaio, R. Neufeld, D. Ferreira, Alginate/chitosan nanoparticles are effective for oral insulin delivery, Pharm. Res. 24 (2007) 2198-2206. https://doi.org/10.1007/s11095-0079367-4

5. A.A. Date, J. Hanes, L.M. Ensign, Nanoparticles for oral delivery: Design, evaluation and state-of-the-art, J. Control. Release 240 (2016) 504-526. https://doi.org/10.1016/j.jconrel.2016.06.016

6. L.M. Ensign, R. Cone, J. Hanes. Oral drug delivery with polymeric nanoparticles: The gastrointestinal mucus barriers, Adv. Drug Deliv. Rev. 64 (2012) 557-570. https://doi.org/10.1016/j.addr.2011.12.009

7. H.K. Han, H.J. Shin, D.H Ha, Improved oral bioavailability of alendronate via mucoadhesive liposomal delivery system, Eur. J. Pharm. Sci. 46 (2012) 500-507. https://doi.org/10.1016/j.ejps.2012.04.002

8. P. Ramalingam, Y.T. Ko, Improved oral delivery of resveratrol from Ntrimethyl chitosan-g-palmitic acid surface-modified solid lipid nanoparticles, Colloid. Surfaces B 139 (2016), 52-61. https://doi.org/10.1016/j.colsurfb.2015.11.050

9. G. Millotti, G. Perera, C. Vigl, K. Pickl, F.M. Sinner, A. BernkopSchnürch, The use of chitosan-6-mercaptonicotinic acid nanoparticles for oral peptide drug delivery, Drug Deliv. 18 (2011), 190-197. https://doi.org/10.3109/10717544.2010.522611

10.M.R. Rekha, C.P. Sharma, Synthesis and evaluation of lauryl succinyl chitosan particles towards oral insulin delivery and absorption, J. Control. Release 135, (2009), 144-151. https://doi.org/10.1016/j.jconrel.2009.01.011

11.B. Tirosh, A. Rubinstein, Migration of adhesive and nonadhesive particles in the rat intestine under altered mucus secretion conditions, J. Pharm. Sci. 87 (1998) 453-456. https://doi.org/10.1021/js9703380

12.K. Maisel, L. Ensign, M. Reddy, R. Cone, J. Hanes, Effect of surface chemistry on nanoparticle interaction with gastrointestinal mucus and distribution in the gastrointestinal tract following oral and rectal administration in the mouse, J. Control. Release 197 (2015) 48-57. https://doi.org/10.1016/j.jconrel.2014.10.026

13.X. Zhu, W. Shan, P. Zhang, Y. Jin, S. Guan, T. Fan, Y. Yang, Z. Zhou, Y. Huang, Penetratin derivative-based nanocomplexes for enhanced intestinal insulin delivery, Mol. Pharm. 11 (2014) 317-328. https://doi.org/10.1021/mp400493b 
14.N. Thirawong, J. Thongborisute, H. Takeuchi, P. Sriamornsak, Improved intestinal absorption of calcitonin by mucoadhesive delivery of novel pectin-liposome nanocomplexes, J. Control. Release 125, (2008) 236-245. https://doi.org/10.1016/j.jconrel.2007.10.023

15.F. Laffleur and A. Bernkop-Schnurch, Thiomers: promising platform for macromolecular drug delivery, Future Med. Chem. 4 (2012) 2205-2216. https://doi.org/10.4155/fmc.12.165

16.S. Dünnhaupt, J. Barthelmes, C.C. Thurner, C. Waldner, D. Sakloetsakun, A. Bernkop-Schnürch, S-protected thiolated chitosan: Synthesis and in vitro characterization. Carbohydr. Polym. 90 (2012), 765-772. https://doi.org/10.1016/j.carbpol.2012.05.028

17.Y. Zambito, F. Felice, A. Fabiano, R. Di Stefano, G. Di Colo, Mucoadhesive nanoparticles made of thiolated quaternary chitosan crosslinked with hyaluronan, Carbohydr. Polym. 92 (2013), 33-39. https://doi.org/10.1016/j.carbpol.2012.09.029

18. Y. Zambito, G. Uccello-Barretta, C. Zaino, F. Balzano, G. Di Colo, Novel transmucosal absorption enhancers obtained by aminoalkylation of chitosan, Eur. J. Pharm. Sci. 29 (2006), 460-469.

https://doi.org/10.1016/j.ejps.2006.09.001

19.H. S. Forrest, J. Walker, Chemotherapeutic agents of the sulphone type. Part V. 2: 5-Disubstituted derivatives of pyridine, J. Chem. Soc. 70 (1948), 1939-1945.

20.S. Dünnhaupt, J. Barthelmes, D. Rahmat, K. Leithner, C.C. Thurner, H. Friedl, A. Bernkop-Schnürch, S-Protected thiolated chitosan for oral delivery of hydrophilic macromolecules: evaluation of permeation enhancing and efflux pump inhibitory properties, Mol. Pharm. 9 (2012), 1331-1341. https://doi.org/10.1021/mp200598j

21.K.E. Constantia, A. Bernkop-Schnürch, Thiolated polymers-thiomers: development and in vitro evaluation of chitosan-thioglycolic acid conjugates, Biomaterials 22 (2001), 2345-2352. https://doi.org/10.1016/S0142-9612(00)00421-X

22.G. Di Colo, Y. Zambito, C. Zaino, M. Sansò, Selected polysaccharides at comparison for their mucoadhesiveness and effect on precorneal residence of different drugs in the rabbit model, Drug Dev. Ind. Pharm. 35 (2009), 941-949. https://doi.org/10.1080/03639040802713460

23.G. Uccello-Barretta, S. Nazzi, F. Balzano, G. Di Colo, Y. Zambito, C. Zaino, M. Sansò, E. Salvadori, M. Benvenuti, Enhanced affinity of ketotifen toward tamarind seed polysaccharide in comparison with hydroxyethylcellulose and hyaluronic acid: a nuclear magnetic resonance investigation, Bioorg. Med. Chem. 16 (2008), 7371-7376. https://doi.org/10.1016/j.bmc.2008.06.020

24. Y. Zambito, E. Pedreschi, G. Di Colo, Is dialysis a reliable method for studying drug release from nanoparticulate systems?-A case study, Int. J. Pharm. 434 (2012), 28-34. https://doi.org/10.1016/j.ijpharm.2012.05.020 
25.A. Fabiano, Y. Zambito, A. Bernkop-Schnürch, About the impact of water movement on the permeation behaviour of nanoparticles in mucus, Int. J. Pharm. 517. (2017), 279-285. https://doi.org/10.1016/j.ijpharm.2016.12.024

26.H. Lennernäs, O. Ahrensted, A. Ungell, Intestinal drug absorption during induced net water absorption in man; a mechanistic study using antipyrine, atenolol and enalaprilat, Brit. J. Clin. Pharmacol. 37 (1994), 589-596.

27.C. Heinen, S. Reuss, S. Saaler-Reinhardt, P. Langguth, Mechanistic basis for unexpected bioavailability enhancement of polyelectrolyte complexes incorporating BCS class III drugs and carrageenans, Eur. J. Pharm. Biopharm. 85 (2013), 26-33. https://doi.org/10.1016/j.ejpb.2013.03.010

28. Y. Zambito, S. Fogli, C. Zaino, F. Stefanelli, M. C. Breschi, G. Di Colo, Synthesis, characterization and evaluation of thiolated quaternary ammonium-chitosan conjugates for enhanced intestinal drug permeation, Eur. J. Pharm. Sci. 38 (2009), 112-120. https://doi.org/10.1016/j.ejps.2009.06.006

29.H. Tozaki, T. Odoriba, N. Okada, T. Fujita, A. Terabe, T. Suzuki, S. Okabe, S. Muranishi, A. Yamamoto, Chitosan capsules for colon-specific drug delivery: enhanced localization of 5-aminosalicylic acid in the large intestine accelerates healing of TNBS-induced colitis in rats, J. Control. Release 82 (2002), 51-61. https://doi.org/10.1016/S0168-3659(02)00084-6

30.Y. Zambito, C. Zaino, G. Uccello-Barretta, F. Balzano, G. Di Colo, Improved synthesis of quaternary ammonium-chitosan conjugates $(\mathrm{N}+-\mathrm{Ch})$ for enhanced intestinal drug permeation, Eur. J. Pharm. Sci. 33 (2008), 343350. https://doi.org/10.1016/j.ejps.2008.01.004

31.P. Jani, G.W. Halbert, J. Langridge, A.T. Florence, Nanoparticle uptake by the rat gastrointestinal mucosa: quantitation and particle size dependency, J. Pharm. Pharmacol. 42 (1990), 821-826. https://doi.org/10.1111/j.20427158.1990.tb07033.x

32.G. Uccello-Barretta, S. Nazzi, Y. Zambito, G. Di Colo, F. Balzano, M. Sansò. Synergistic interaction between TS-polysaccharide and hyaluronic acid: implications in the formulation of eye drops, Int. J. Pharm. 395 (2010):122-131. https://doi.org/10.1016/j.ijpharm.2010.05.031

33.F. Laffleur, A. Fischer, M. Schmutzler, F. Hintzen, A. Bernkop-Schnürch, Evaluation of functional characteristics of preactivated thiolated chitosan as potential therapeutic agent for dry mouth syndrome, Acta Biomater. 21 (2015), 123-131. https://doi.org/10.1016/j.actbio.2015.04.016

34.Legen, S. Mateja, K. Janez, Comparison of different intestinal epithelia as models for absorption enhancement studies, Int. J. Pharm. 291 (2005), 183188.

35.G. Di Colo, Y. Zambito, C. Zaino, Polymeric enhancers of mucosal epithelia permeability: synthesis, transepithelial penetration-enhancing properties, mechanism of action, safety issues, J. Pharm. Sci 97 (2008), 1652-1680. https://doi.org/10.1002/jps.21043 
36.S. Nagayama, K. Ogawara, Y. Fukuoka, K. Higaki, T. Kimura, Timedependent changes in opsonin amount associated on nanoparticles alter their hepatic uptake characteristics, Int. J. Pharm. 342 (2007), 215-221. https://doi.org/10.1016/j.ijpharm.2007.04.036

37.Y.N. Zhang, W. Poon, A.J. Tavares, I.D. Mc Gilvray, W.C.W. Chan, Nanoparticle-liver interactions: cellular uptake and hepatobiliary elimination, J. Control. Release 240 (2016), 332-348. https://doi.org/10.1016/j.jconrel.2016.01.020

38.R.K. Anderson, S.J. Fogelson, The secretion of gastric mucin in man. A comparative study in the normal subject and in the patient with peptic ulcer in response to an alcohol test, J. Clin. Invest. 15 (1936), 169-172. https://doi.org/10.1172/JCI100765

39.A. Fabiano, P. Chetoni, Y. Zambito, Mucoadhesive nano-sized supramolecular assemblies for improved pre-corneal drug residence time, Drug Dev. Ind. Pharm. 41 (2015), 2068-2076. https://doi.org/10.3109/03639045.2015.1066798 
Table 1. Characteristics of QA-Ch-SH and QA-Ch-S-pro: degree of substitution $(\mathrm{DS} \% \pm \mathrm{SD}$ ) on a polymer repeating unit basis.

\begin{tabular}{|l|l|l|l|}
\hline Polymer & $\begin{array}{l}\text { Thiol-bearing } \\
\text { groups }^{\text {a }} \%\end{array}$ & Free thiols, $\%$ & Protected thiols, \% \\
\hline QA-Ch-SH & $16 \pm 6$ & $1.0 \pm 0.0$ & - \\
\hline QA-CH-S-pro & $16 \pm 6$ & $0.4 \pm 0.2$ & $1.9 \pm 0.2$ \\
\hline
\end{tabular}

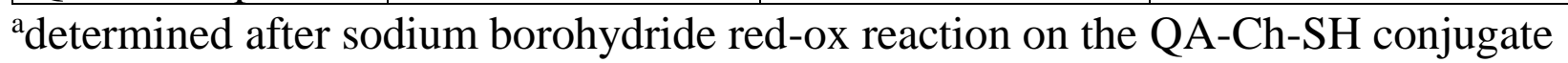


Table 2. Characteristics of NP determined after purification. Mean $\pm \operatorname{SD}(\mathrm{n}=3)$.

\begin{tabular}{|l|l|l|l|l|}
\hline NP type & $\begin{array}{l}\text { Nanoparticle size, } \mathrm{nm} \\
\text { (polydispersity index) }\end{array}$ & $\zeta, \mathrm{mV}$ & $\mathrm{EE}, \%$ & $\begin{array}{l}\mathrm{DRD}^{\mathrm{a}}, \\
\mathrm{mg} / \mathrm{ml}\end{array}$ \\
\hline NP QA-Ch & $371 \pm 38(0.4 \pm 0.1)$ & $13 \pm 1$ & $23 \pm 5$ & 0.14 \\
\hline NP QA-Ch-S-pro & $376 \pm 82(0.4 \pm 0.1)$ & $12 \pm 1$ & $23 \pm 8$ & 0.14 \\
\hline
\end{tabular}

${ }^{\text {ad }}$ rug concentration in regenerated dispersion 
Table 3. Mass fraction adsorbed on rat intestinal mucosa from FD4 solution (control), NP QA-Ch dispersion, or NP QA-Ch-S-pro dispersion. Means \pm SD $(n=3)$. Data marked by * are significantly different from the control FD4; the data marked by $* *$ are significantly different from either of the others.

\begin{tabular}{|l|l|l|}
\hline Formulation & $\begin{array}{l}\text { Adsorbed mass fraction, } \\
\%\end{array}$ & TR \\
\hline FD4 & $12 \pm 2$ & 0.90 \\
\hline NP QA-Ch & $23 \pm 3 *$ & 0.75 \\
\hline NP QA-Ch-S-pro & $45 \pm 2 * *$ & 0.37 \\
\hline
\end{tabular}


Table 4. Data on FD4 apparent permeation across excised rat intestine.

\begin{tabular}{|l|l|l|l|l|}
\hline Formulation & $\begin{array}{l}\text { Flux } 10^{2}(\mu \mathrm{g} \\
\left.\mathrm{cm}^{-2} \mathrm{~min}^{-1}\right)\end{array}$ & $\begin{array}{l}\mathrm{P}_{\text {app }} 10^{5 \mathrm{a}}(\mathrm{cm} \\
\left.\mathrm{sec}^{-1}\right)\end{array}$ & $\mathrm{EPR}^{\mathrm{b}}$ & $\mathrm{T}_{4 \mathrm{~h}}{ }^{\mathrm{c}}\left(\mu \mathrm{g} \mathrm{cm}{ }^{-2}\right)$ \\
\hline FD4 & $0.44 \pm 0.04$ & $4.4 \pm 0.4$ & - & $1.2 \pm 0.1$ \\
\hline NP QA-Ch & $0.88 \pm 0.08^{*}$ & $8.8 \pm 0.8^{*}$ & 2.0 & $2.2 \pm 0.1^{*}$ \\
\hline NP QA-Ch-S-pro & $1.07 \pm 0.01^{*}$ & $10.1 \pm 1.1^{*}$ & 2.3 & $2.0 \pm 0.2^{*}$ \\
\hline
\end{tabular}

${ }^{a}$ Apparent permeability.

${ }^{\mathrm{b}}$ Enhanced permeability ratio (ratio of $\mathrm{P}_{\mathrm{app}}$ to the value for the control FD4).

${ }^{\mathrm{c}}$ Cumulative transport over the whole time of experiment (4h).

*Significantly different from the control FD4 . 
Table 5. Pharmacokinetic parameters after oral administration of FD4, NP QA-Ch and NP QA-Ch-S-pro. The data marked by * is significantly different from the control FD4. The data marked by $* *$ is significantly different from either of the others.

\begin{tabular}{|l|l|l|l|l|}
\hline Formulation & $\mathrm{C}_{\max }(\mu \mathrm{g} / \mathrm{mL})$ & $\mathrm{T}_{\max }(\mathrm{h})$ & $\mathrm{AUC}_{0-5}(\mu \mathrm{g} / \mathrm{mL} \min )$ & $\mathrm{AUC}_{\text {rel }}$ \\
\hline FD4 & $0.38 \pm 0.13$ & 1 & $1.25 \pm 0.13$ & - \\
\hline NP QA-Ch & $0.67 \pm 0.11^{*}$ & 1 & $1.71 \pm 0.26^{*}$ & 1.4 \\
\hline NP QA-Ch-S-pro & $0.91 \pm 0.03^{*}$ & 2 & $2.91 \pm 0.36^{* *}$ & 2.4 \\
\hline
\end{tabular}



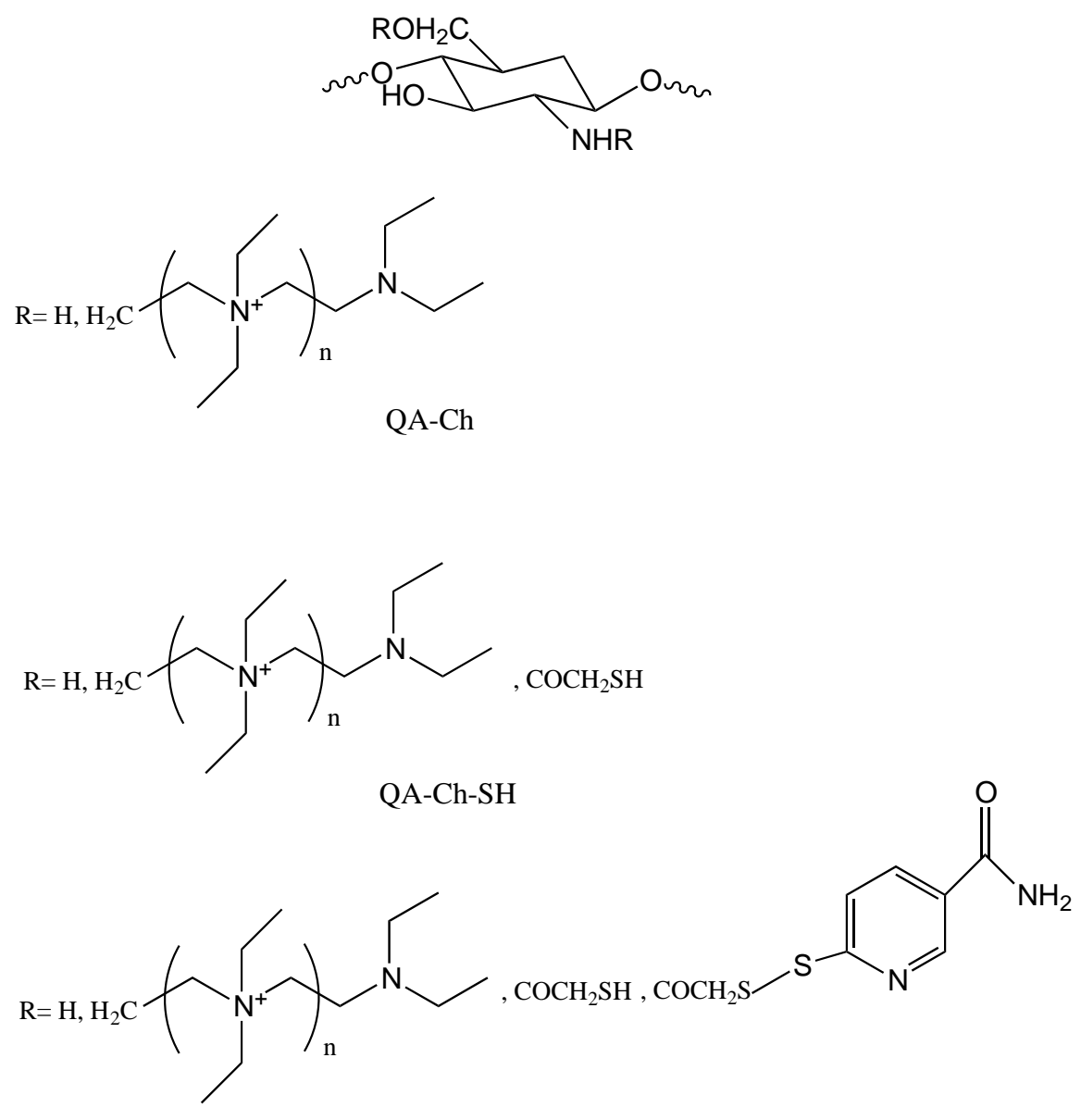

QA-Ch-S-pro

Figure 1. Structural characteristics of QA-Ch, QA-Ch-SH and QA-Ch-S-pro. 


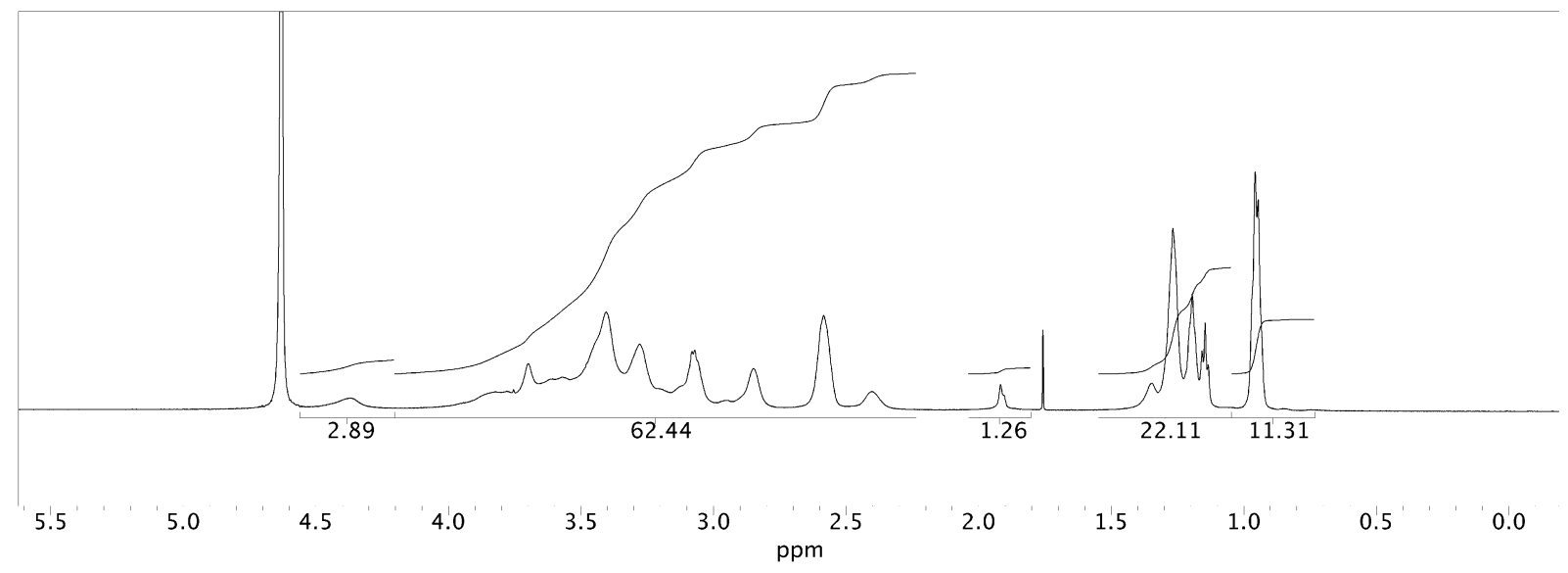

Figure 2. ${ }^{1} \mathrm{H}$ NMR $\left(600 \mathrm{Mhz}, \mathrm{PB} \mathrm{D}_{2} \mathrm{O}, 25 * \mathrm{C}\right)$ spectrum of QA-Ch with integral values. 


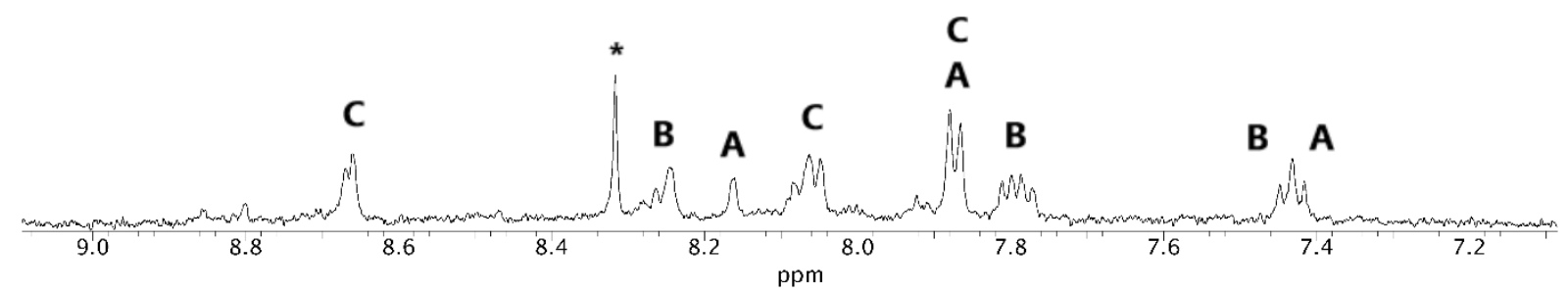

Figure 3. ${ }^{1} \mathrm{H}$ NMR $\left(600 \mathrm{Mhz}, \mathrm{PB}_{2} \mathrm{O}, 25 * \mathrm{C}\right)$ spectral region between $7.5 \div 9.0$ of QA-Ch-S-pro (* was assigned to unknown impurity). 


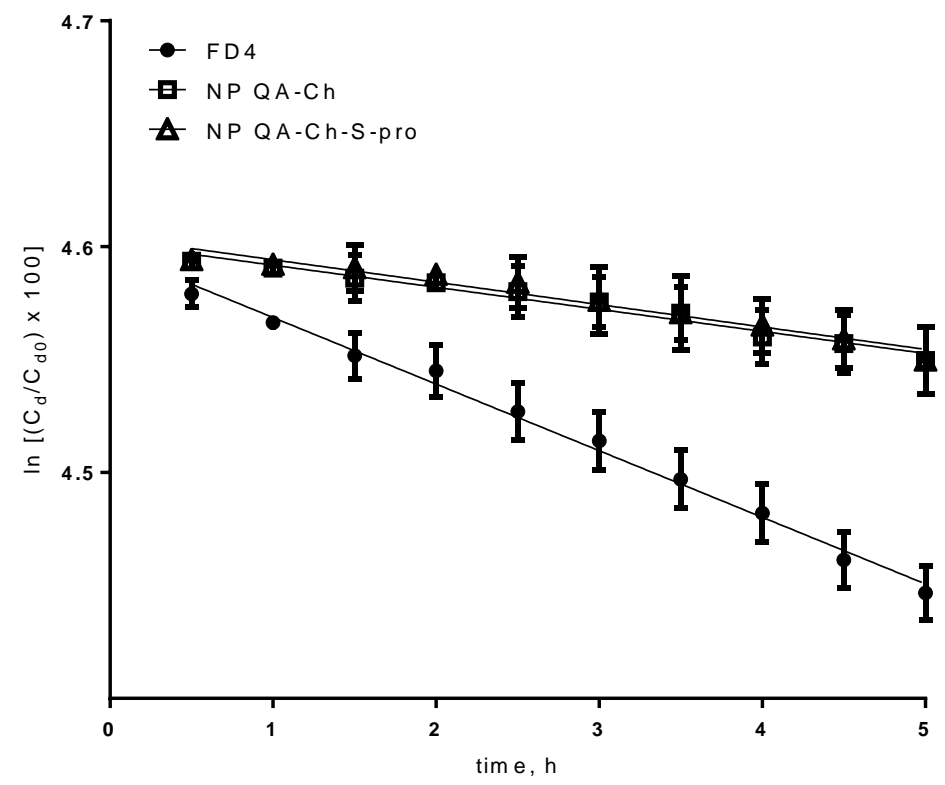

Figure 4. Dynamic dialysis data plotted according to Equation 1. Means \pm SD $(n=3-6)$. 

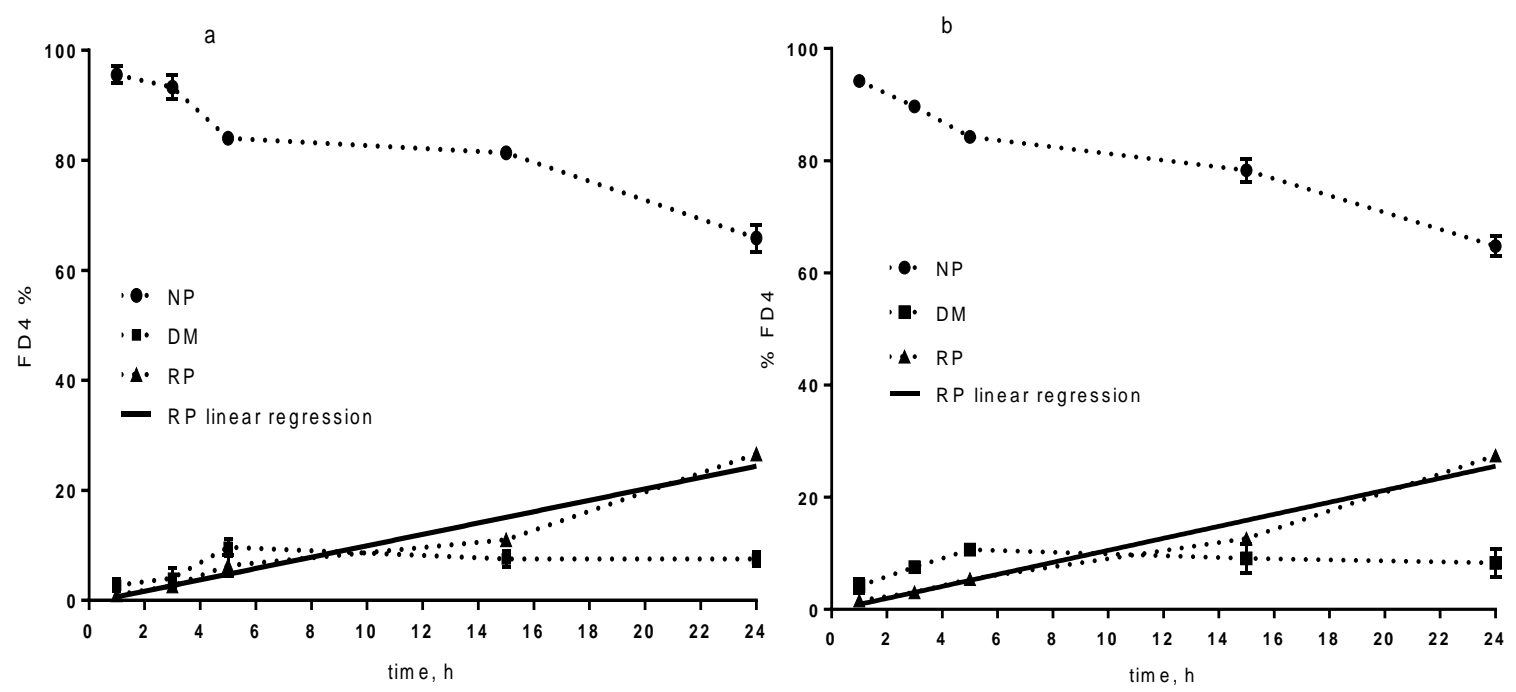

Figure 5. Interrupted dialysis studies: kinetic analysis of dialysis phases for FD4loaded QA-Ch (A) and QA-Ch-S-pro (B) NP regenerated after lyiophilization. $\mathrm{NP}=\mathrm{NP}$ matrix; $\mathrm{DM}=\mathrm{NP}$ dispersion medium; $\mathrm{RP}=$ receiving phase. Means $\pm \mathrm{SD}$ $(n=3)$. 


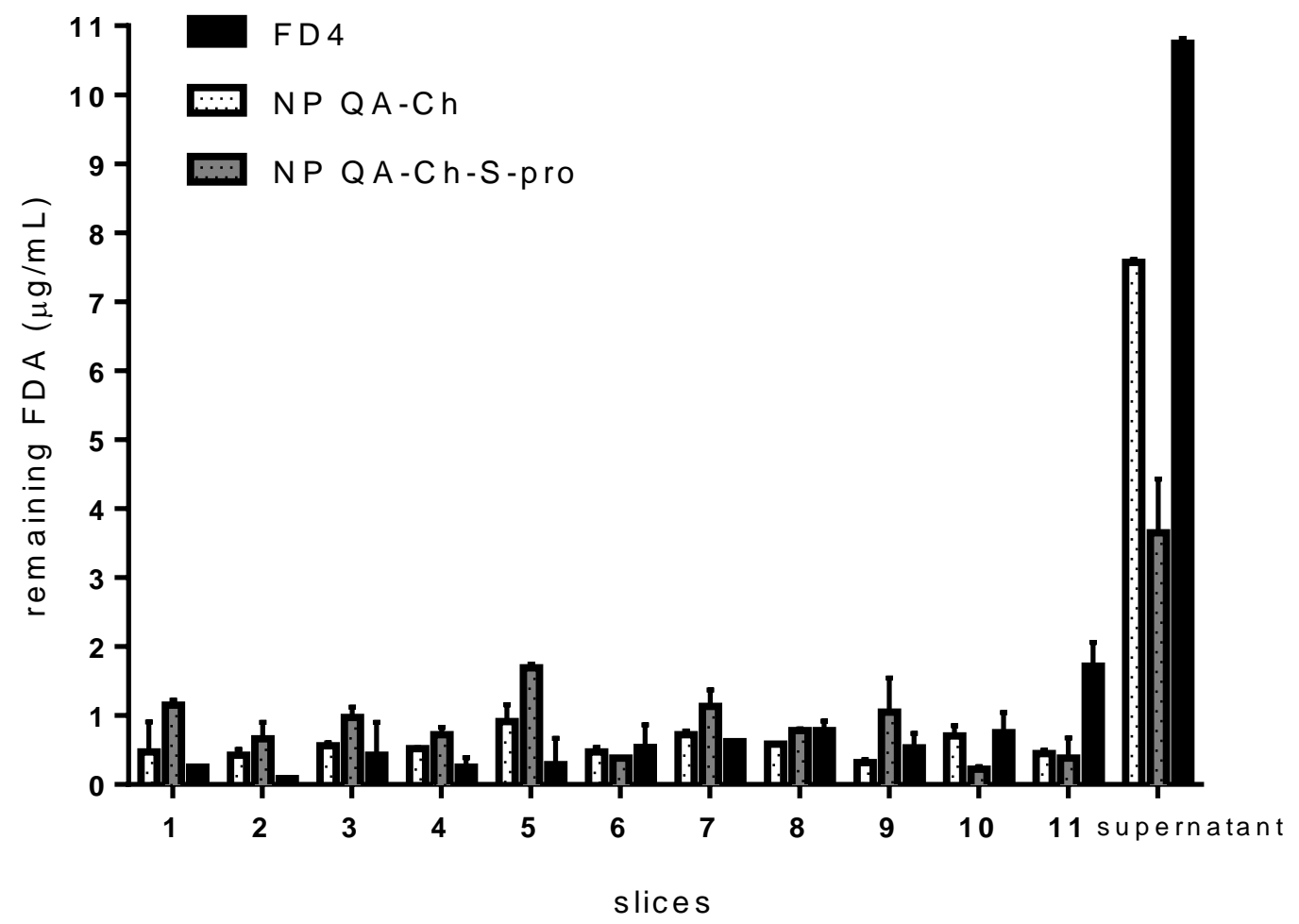

Figure 6. Water-assisted transport in mucus of FD4-loaded QA-Ch and QA-Ch-Spro NP regenerated after lyophilization. Means $\pm \mathrm{SD}(\mathrm{n}=3)$. 


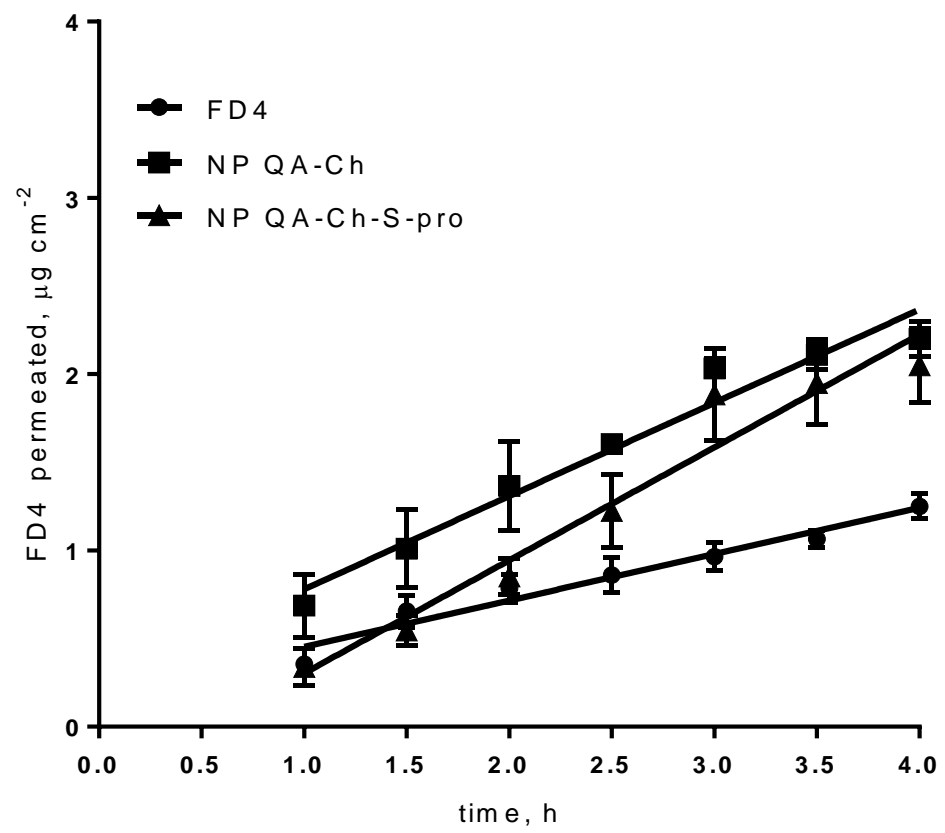

Figure 7. FD4 apparent permeation plots across excised rat intestine. Means \pm SD $(n=3-6)$. 


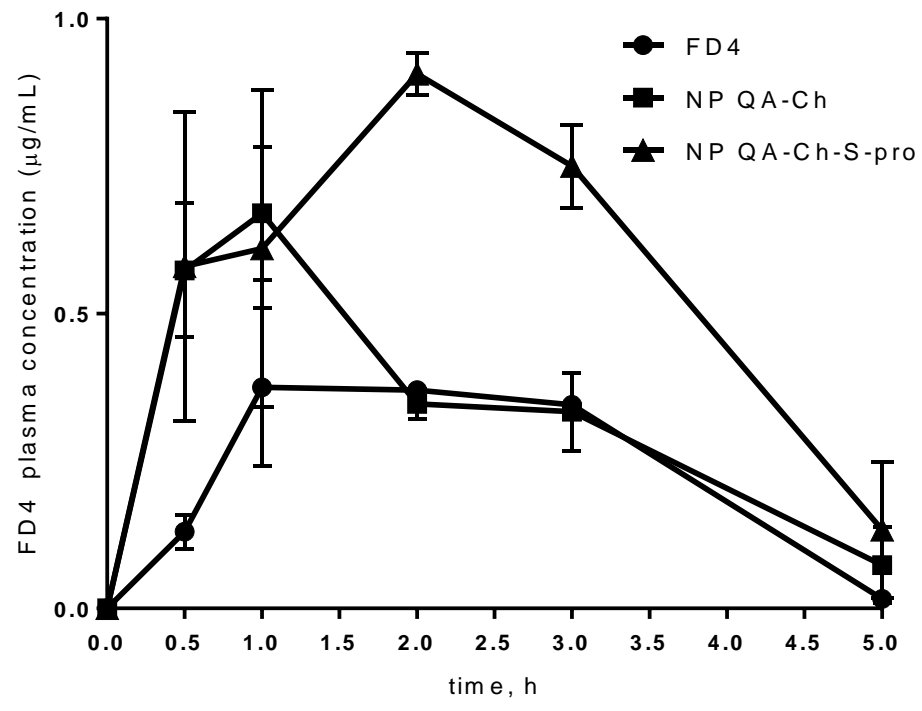

Figure 8. FD4 plasma-concentration-time profiles after administration of NP QA$\mathrm{Ch}$ and NP QA-Ch-S-pro in comparison with the control FD4. Means \pm SD $(n=3)$. 


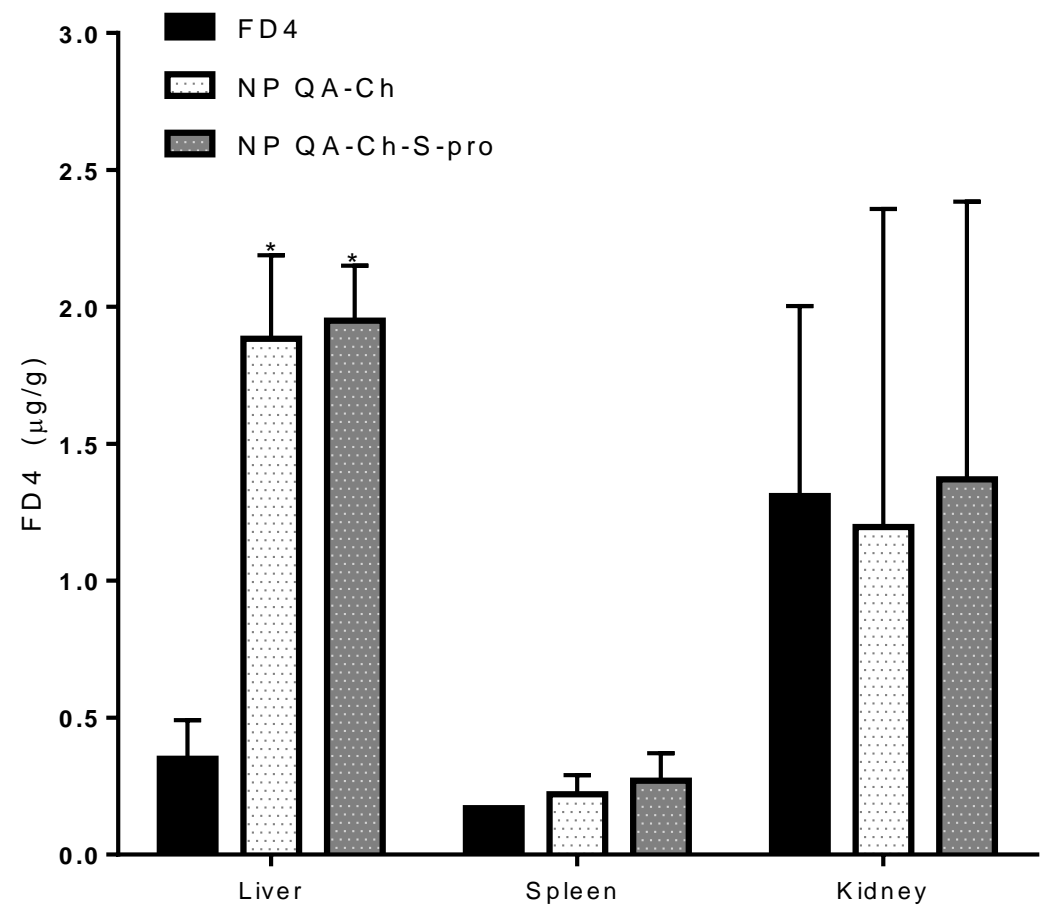

Figure 9. Quantity $(\mu \mathrm{g})$ of FD4 per gram of organ withdrawn at the end of the experiment $(5 \mathrm{~h})$. Means $\pm \mathrm{SD}(\mathrm{n}=3)$. The data marked by $*$ are significantly different from the control. 
Supplemental File

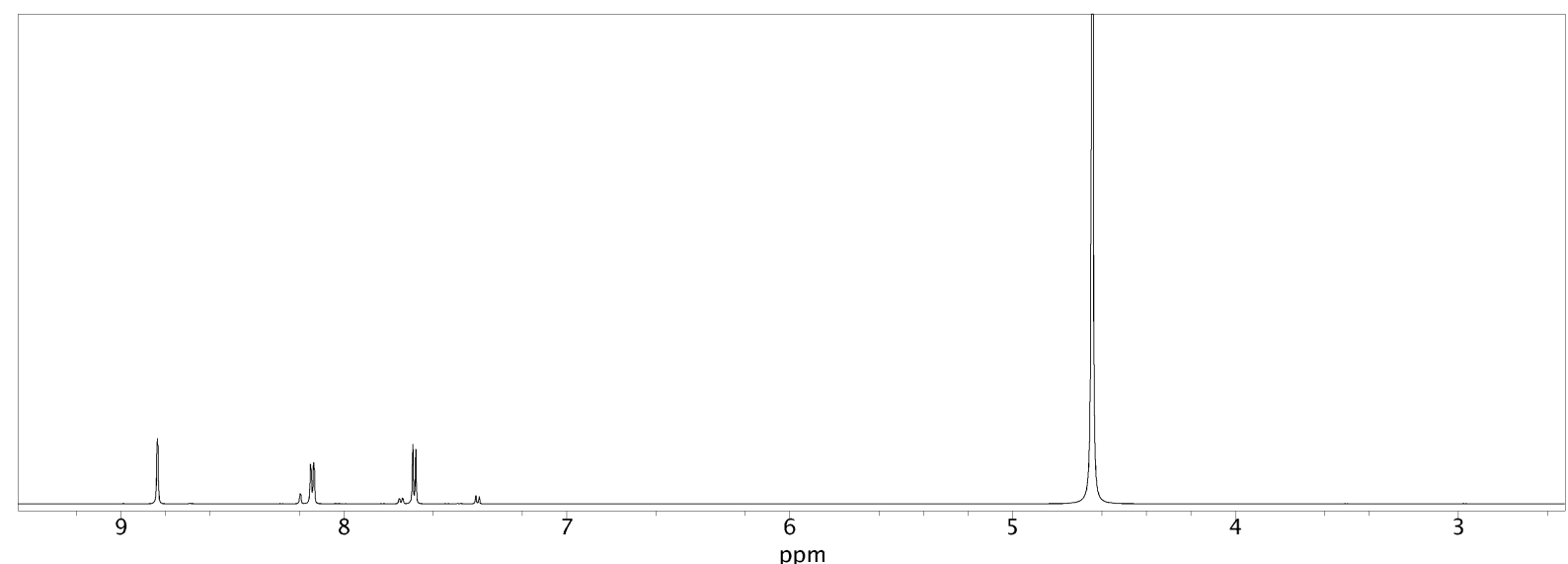

Figure S1. ${ }^{1} \mathrm{H}$ NMR $(600 \mathrm{Mhz}, \mathrm{DMSO}, 25 * \mathrm{C})$ of Cl-NA. 


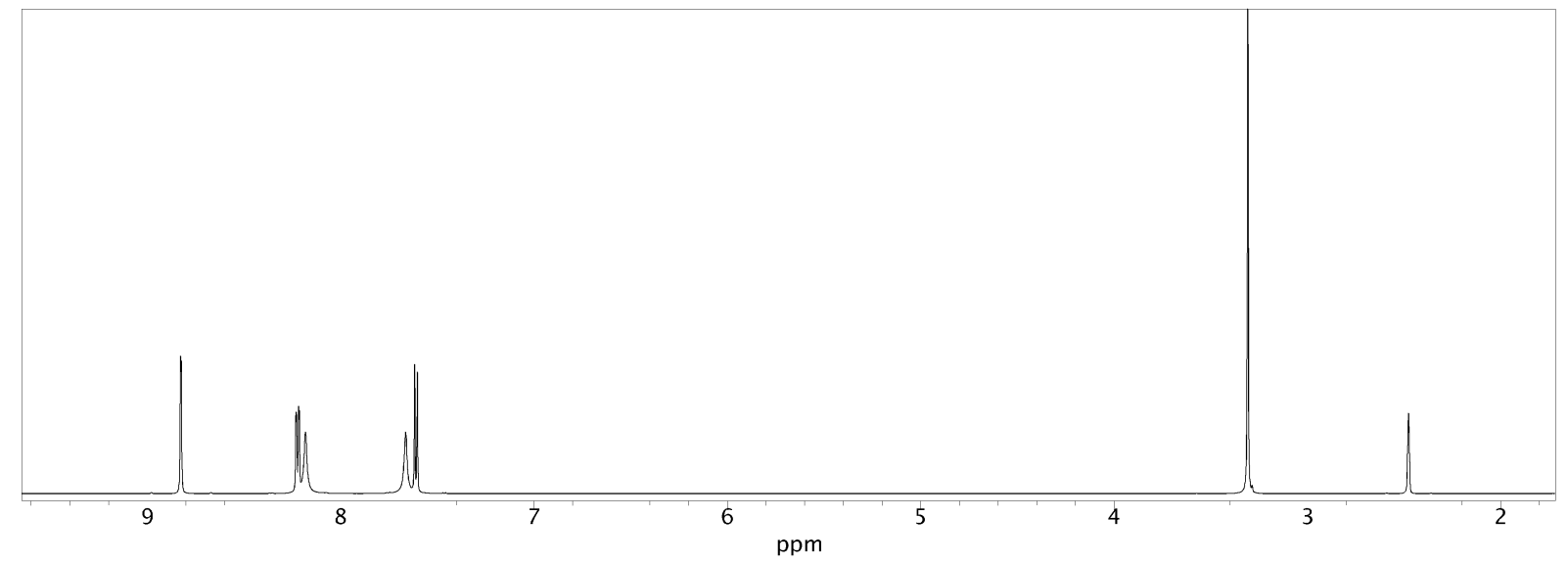

Figure S2. ${ }^{1} \mathrm{H}$ NMR $\left(600 \mathrm{Mhz}, \mathrm{PB}_{2} \mathrm{O}, 25{ }^{*} \mathrm{C}\right)$ of isothiouronium salt of nicotinamide. 


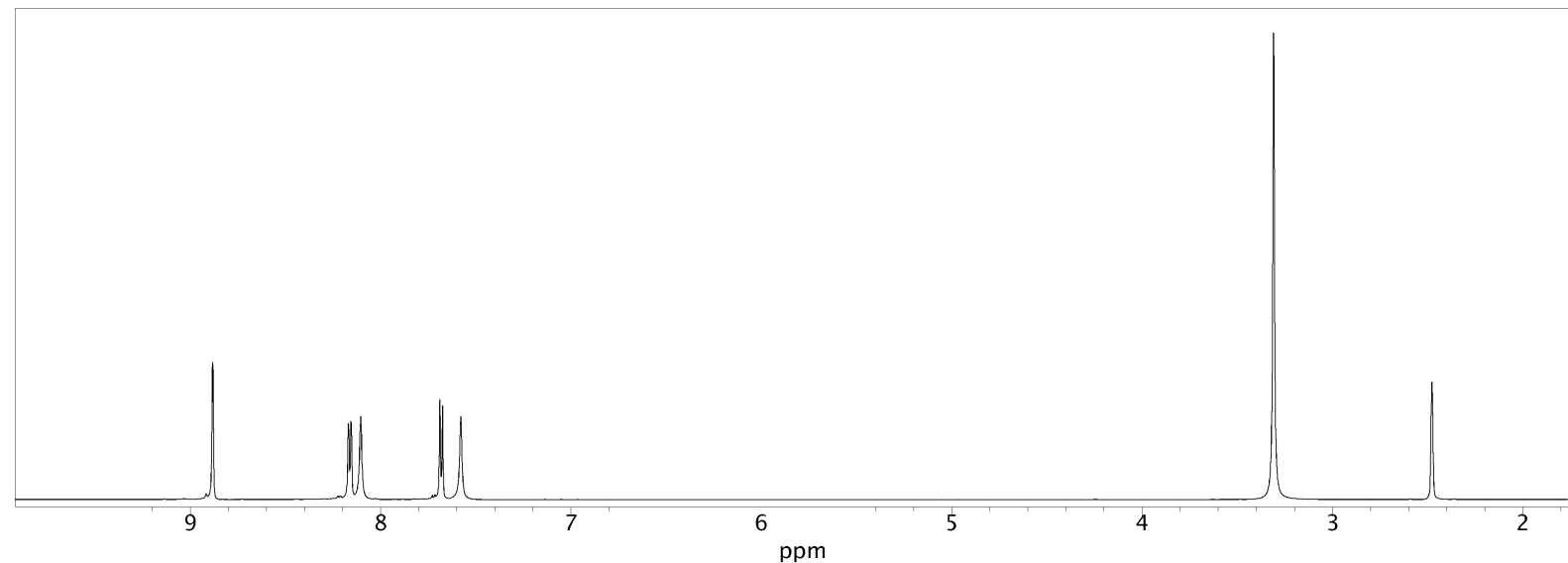

Figure S3. ${ }^{1} \mathrm{H}$ NMR $\left(600 \mathrm{Mhz}, \mathrm{DMSO}, 25{ }^{*} \mathrm{C}\right)$ of 6,6 '-DTNA. 


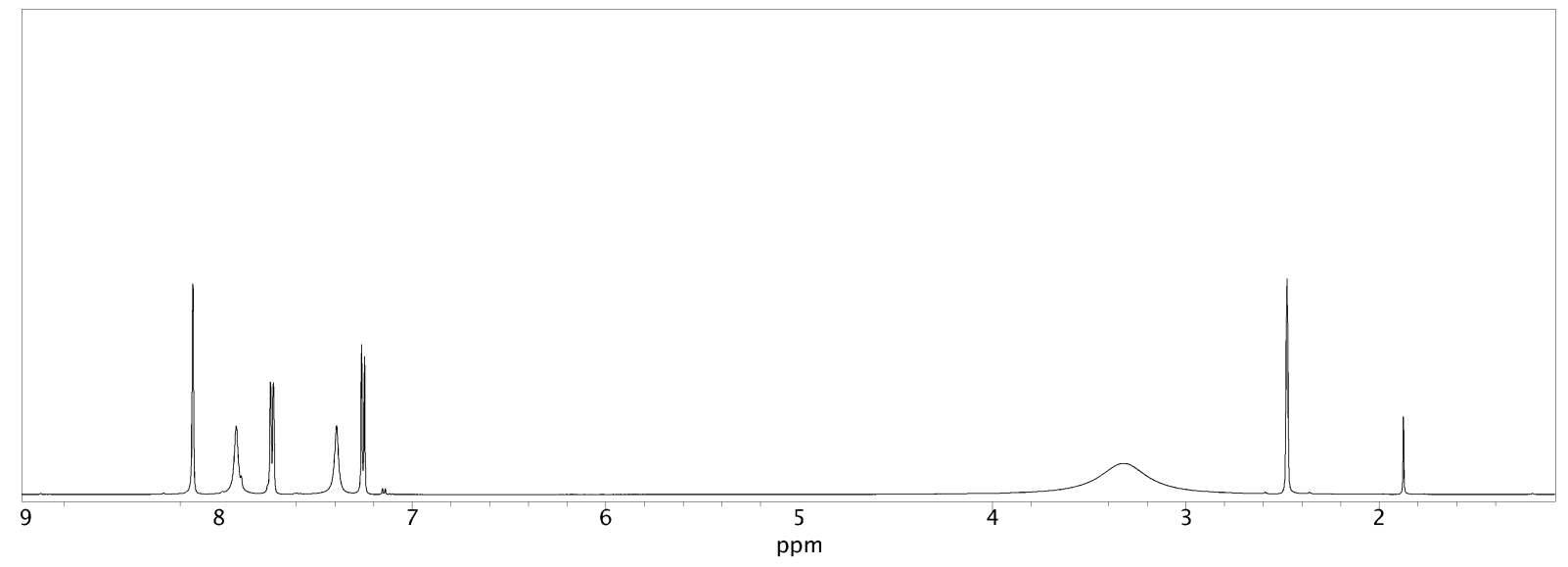

Figure S4. ${ }^{1} \mathrm{H}$ NMR $\left(600 \mathrm{Mhz}, \mathrm{DMSO}, 25{ }^{*} \mathrm{C}\right)$ of 6-MNA. 


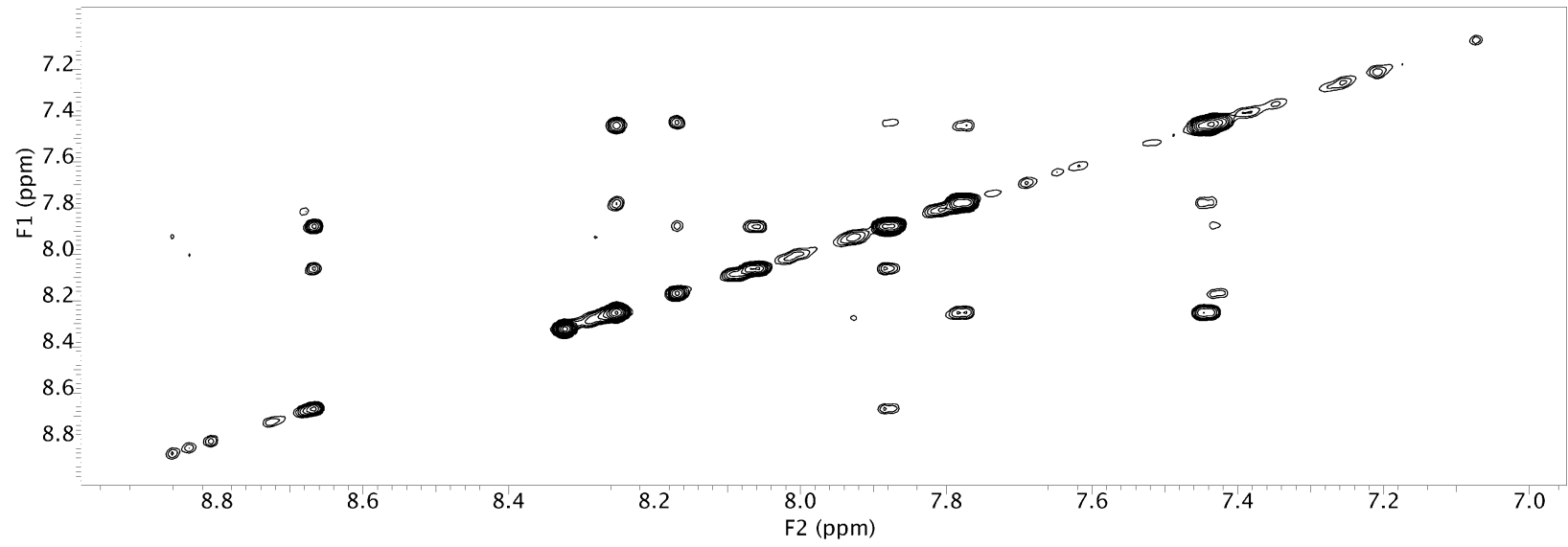

Figure S5. TOCSY $\left(600 \mathrm{Mhz}, \mathrm{PB}_{2} \mathrm{O}, 25 * \mathrm{C}\right)$ spectral region between $7.5 \div 9.0$ of QA-Ch-S-Pro 